\title{
Modeling the eye as a window on the body
}

Lucia Carichino ${ }^{1}$, Simone Cassani², Sergey Lapin ${ }^{3}$, Alice Verticchio Vercellin ${ }^{4-6}$

${ }^{1}$ School of Mathematical Sciences, Rochester Institute of Technology, Rochester, NY, USA; ${ }^{2}$ Department of Mathematics, University at Buffalo, SUNY, Buffalo, NY, USA; ${ }^{3}$ Department of Mathematics and Statistics, Washington State University, Pullman, Washington, USA; ${ }^{4}$ University of Pavia, Pavia, Italy; ${ }^{5}$ RCCS - Fondazione Bietti, Rome, Italy; ${ }^{6}$ Department of Ophthalmology, Icahn School of Medicine at Mount Sinai Hospital, New York, NY, USA

Keywords: mathematical modeling, multidisciplinary approach, ocular and systemic diseases

\section{Introduction}

Systemic pathologies such as diabetes and arterial hypertension affect different organs and systems in the body. ${ }^{1-4}$ However, the first signs of these pathologies often emerge as functional and structural alterations in the eye..$^{1-4}$ As a consequence, the ophthalmologist is often the first physician to make a diagnosis of systemic diseases. In fact, the eye represents a unique organ where signs of systemic diseases may be assessed with noninvasive techniques. ${ }^{3}$ The vessels of the retinal microcirculation are the only ones in the whole body where the physician, via the examination of the fundus oculi, can observe vessel health directly and noninvasively. In this sense, the diagnosis and follow-up of important systemic conditions, such as diabetes and arterial hypertension, is performed by the periodical examination of the fundus oculi. The assessment of visual acuity is a tool for early diagnosis of diabetes since high glucose levels in the aqueous humor, the fluid filling the anterior and posterior chamber of the eye, are correlated to a myopic shift in the visual acuity. The visual field examination allows to assess both ophthalmological diseases, such as glaucoma, and neurological ones, such as brain tumors, cerebral ischemia, or multiples sclerosis, of which the most common first manifestation is a retrobulbar optic neuritis ${ }^{3}$. If not promptly diagnosed and treated, the complications of the aforementioned diseases - such as cardiovascular disorders, nephropathy, and neuropathies - are potentially

Correspondence: Lucia Carichino, Rochester Institute of Technology, School of Mathematical Sciences, 85 Lomb Memorial Drive, Rochester, NY 14623, USA.

E-mail: Icsma1@rit.edu 
harmful for the health of the individual. Therefore, the eye examination is important to make an early diagnosis of such diseases and to prevent clinical complications.

Mathematical modeling represents an important tool that can help in the analysis, early diagnosis, and treatment of systemic and ocular diseases. ${ }^{5-8}$ In order to build mathematical models that can work in synergy with clinical data, it is important to create active interdisciplinary collaborations between experts in different mathematical areas and medical professionals. The workshop "Modeling the eye as a window on the body" that was held in San Jose, CA, USA October 15-19 2018, brought together experts in mathematical modeling of the eye and other organs in the body, medical doctors, statisticians, and biomedical engineers to share their perspectives and expertise with the final goal to develop mathematical models linking the eye to the body.

Mathematical models represent a virtual lab that can be used to:

1. elucidate the driving mechanisms leading to a pathology;

2. isolate and quantify the relative contribution of factors that cannot be separated in vivo; and

3. test clinical hypotheses.

To date, only preliminary mathematical models linking ocular dynamics with the cardiovascular system and the dynamics in other organs, such as the kidney and brain, are available. ${ }^{9}$ Several mathematical models studying systems separately have been previously developed; $6^{6-8}$ however, the coupling of the different vascular and structural components involved introduces multiple challenges. Specifically, the coupling involves the following multiscale and multiphysics components: fluid flows (e.g. aqueous humor, blood, and urine), structural deformations, oxygen transport, pressurized ambients, local vascular regulatory mechanisms, and micro- and macro-vasculature networks. This will require sophisticated mathematical techniques such as systems of mixed hyperbolic, parabolic, and elliptic partial differential equations involving multiple time scales. Therefore, this project endeavors active collaborations among experts in different mathematical areas, such as differential equations, fluid structure interaction, reduced and compartmental models, and numerical analysis. Since the condition of the eye is indicative of the one of other organs, it is important to define coupled mathematical models that can correlate and/or predict the effect of different diseases in different anatomical regions.

Statistical analysis and clinical data are essential to consolidate model predictions. In medicine, statistical analysis is one of the most common uses of mathematics to extrapolate significant correlations in the data. Moreover, statistical analysis, mathematical models, and clinical data can be used in synergy to build reliable and accurate models by parameter estimation, to validate model results with data, and to elucidate the mechanisms behind clinical correlations. ${ }^{10-13}$ The Structured Quartet Research Ensemble (SQuaRE) on "Ocular blood flow and its role in development of glaucoma", held at The American Institute of Mathematics (AIM) in 2017, is an example of how an integrative collaboration between mathematics, ophthalmology, and statistics can 
provide better medical care for patients with various complicated diseases, such as glaucoma. The results of this collaborative effort have been published in scientific and clinical journals. ${ }^{11-12}$

\section{Workshop "Modeling the eye as a window on the body"}

The workshop "Modeling the eye as a window on the body" brought together twenty participants among leading experts and new researchers (young faculty members, postdoctoral, and graduate students) from universities in the United States, Canada, Great Britain, Italy and France, with the following common goals:

1. Provide a forum to discuss how to address the challenges of the mathematical coupling of the eye to the rest of the body.

2. Exchange ideas on the potential of using mathematical models of the eye to:

- Get insights on the effects of aging and systemic pathologies, such as arterial hypertension and diabetes, on the ocular structure and functionality.

- Achieve a better understanding of the processes that regulate the level of intraocular pressure (IOP) and oxygenation in the retinal tissue.

3. Identify the clinical questions of interest for the coupled mathematical models under investigation.

To achieve these goals, the participants attended lectures in the morning and participated in group activities in the afternoon focused on specific topics. Of note, the groups were organized in such a way that each one contained at least one expert in medicine.

\section{Workshop lectures}

The workshop lectures aimed to discuss the current state of the art and open problems from various points of view: clinical, mathematical, statistical, and engineering. Among the notable lectures given by the workshop participants, we list a number of them below.

From the clinical perspective, Carlo Bruttini (University of Pavia, Pavia, Italy) presented a review of the relationship between the eye and vascular systemic pathologies, with a special focus on diabetic retinopathy, ${ }^{1,3}$ thrombosis and occlusions of ocular vessels, ${ }^{3}$ and the effect of high altitude on the production and drainage of aqueous humor, ${ }^{14}$ which regulates IOP.

From the mathematical perspective, Giovanna Guidoboni (University of Missouri, Columbia, MO, USA) discussed the state of the art of modeling in ophthalmology, focusing on mathematical models developed to study ocular hemodynamics, ocular structural deformation, aqueous humor production, and retinal oxygenation. ${ }^{5}$ 
The mathematical models presented have been used in synergy with clinical data to interpret medical measurements, and to try to isolate the different pathogenic mechanisms that could lead to the specific results observed in each individual patient. Anita Layton (University of Waterloo, Waterloo, Canada) presented multiscale mathematical models of kidney physiology and pathophysiology to study the role of the kidney in regulating blood flow and blood pressure. ${ }^{15}$ In particular, the presentation focused on the role played by mathematical models in the understanding of the mechanisms underlying any potential cardiovascular benefit of kidney-targeting drugs for diabetes. ${ }^{16}$

From the engineering perspective, Giovanni Ometto (City, University of London, London, United Kingdom) presented recent work on image processing of retinal fundus photographs. The image-processing algorithm developed was used to detect positions of microaneurisms in diabetic patients. The algorithm, in synergy with statistical analysis, was employed to identify the area where lesions could predict progression to vision-threatening diabetic retinopathy. ${ }^{17}$

From the statistical perspective, Yuan Wang (Washington State University, Pullman, WA, USA) presented results on statistical analysis techniques to study tree-structured data, focusing on applications related to the brain artery tree. ${ }^{18}$ Andrea Arnold (Worcester Polytechnic Institute, Worcester, MA, USA) presented a review of the Bayesian approach for state and parameter estimation using nonlinear Kalman filtering and data. ${ }^{19}$

\section{Future research directions}

As a result of the workshop lectures and group discussions, the participants identified the following topics of interest for future research. Each topic includes challenges from the mathematical perspective while addressing questions of significant clinical relevance.

\subsection{Aqueous humor physiology and IOP}

Aqueous humor, the fluid filling the anterior and posterior chamber of the eye, has many important functions, among which is the regulation of IOP. ${ }^{3}$ Aqueous humor production involves a filtration process from the fenestrated capillaries of the ciliary body. This process is similar to the kidney nephron glomerular filtration. ${ }^{22}$ Hence, the idea is to develop a compartmental model of aqueous humor production inspired by existing kidney models that could account for the effects of IOP-lowering medications with several mechanisms of action on the different ion channels. The preliminary results of this collaboration were presented as poster at the 2019 Association for Research in Vision and Ophthalmology (ARVO) Annual Meeting in Vancouver, Canada. ${ }^{23}$ 
L. Carichino, S. Cassani and S. Lapin et al.

\subsection{Neovascularization in wet age-related macular degeneration (AMD)}

AMD neovascularization is responsible for severe vision loss, ${ }^{3}$ and modeling this phenomenon involves sophisticated multiphase and multiscale modeling techniques. Only a few mathematical models of AMD choroidal neovascularization are available in the literature. ${ }^{20-21}$ The participants discussed the importance of developing a model that includes the effect of anti-vascular endothelial growth factor (anti-VEGF) treatments on the neovascularization growth. The model will then be used in synergy with clinical images of choroidal neovascularization, acquired with optical coherence tomography (OCT) and OCT angiography, to investigate the mechanisms behind the differences in anti-VEGF treatment efficiency in individual patients.

\subsection{Age and retrobulbar blood flow}

Color Doppler imaging (CDI) is a common imaging technique used to assess the blood flow velocities in several vessels in the body. Specifically in the eye, blood flow velocities are assessed in the retrobulbar vessels (ophthalmic artery, central retinal artery, and posterior ciliary arteries) and the participants shared their knowledge about the hemodynamic changes in glaucoma. ${ }^{24}$ In the future, the use of $\mathrm{CDI}$ to assess and compare blood flow in the retrobulbar circulation and in other parts of the body in both elderly and young subjects can further the understanding of the role of aging in the pathophysiology of ocular and systemic diseases.

\subsection{Oxygenation in light and darkness}

It has been reported in the clinical literature that retinal oxygen saturation is higher in dark than light in healthy humans. ${ }^{25}$ This result is conjectured to be the effect of different changes in the metabolic consumption of the tissue in the inner and outer retina in dark and light. To explore this hypothesis, the participants utilized a coupled model of the retinal vasculature and retinal tissue oxygen saturation. ${ }^{26}$ The model was used to obtain preliminary results relative to the effect of changes in the metabolic consumption of the different retinal tissue layers on the level of retinal oxygen saturation. The model will be further improved and used to determine the underlying mechanisms governing oxygen saturation and tissue consumption in the retinal tissue to achieve a better understanding of the results recorded in the clinical literature.

\subsection{Imaging predictors of diabetes progression}

A number of imaging techniques, such as fluorescein angiography and $\mathrm{OCT},{ }^{1}$ are currently used to evaluate retinal lesions due to diabetic retinopathy. The aforementioned imaging tools are used for early diagnosis of diabetic retinopathy, and to detect and predict the disease progression. The participants discussed the potential use of $\mathrm{CDI}$ and $\mathrm{CDI}$-derived parameters previously used for glaucoma (i.e. velocity waveform parameters in the ophthalmic artery, central retinal artery, and posterior 
ciliary arteries), for the assessment and early detection of disease progression in patients with diabetic retinopathy.

\section{Conclusions}

A fruitful collaboration between mathematicians and medical professionals can significantly contribute to both better mathematical descriptions of human physiology and improved medical procedures. In this context, the workshop "Modeling the eye as a window on the body" led to the creation of an international network of experts in different fields that will continue to collaborate to further the development of medical and mathematical research, and to pursue multiple directions for future research in different fields. The insights coming from this interdisciplinary collaboration may ultimately aid the future development of new techniques and instruments designed by biomedical engineers for the diagnosis and treatment of systemic and ophthalmological pathologies.

\section{Acknowledgements}

The authors would like to thank the American Institute of Mathematics (AIM) staff and all the participants of the workshop "Modeling the eye as a window on the body" for their help and support.

The authors have no financial or proprietary interest in any material or method mentioned. The contribution of the author Dr. Alice C. Verticchio Vercellin was supported by Fondazione Roma and by the Italian Ministry of Health.

\section{References}

1. Shotliff K, Duncan G. Diabetes and the eye. In: KM Shaw, MH Cummings, editors. Diabetes: Chronic Complications. Chichester: John Wiley and Sons; 2005.

2. Konstantinidis L, Guex-Crosier Y. Hypertension and the eye. Curr Opin Ophthalmol. 2016;27(6):514521.

3. Kansky JJ. Clinical ophthalmology: A systemic approach. 8th edition. Elsevier; 2015.

4. Flammer J, Konieczka K, Bruno R, Virdis A, Flammer AJ, Taddei S. The eye and the heart. Eur Heart J. 2013;34(17):1270-1278.

5. Gross JC, Harris A, Siesky BA, Sacco R, Shah A, Guidoboni G. Mathematical modeling for novel treatment approaches to open-angle glaucoma. Exp Rev Ophthalmol. 2017;(12)6:443-455.

6. Guidoboni G., Harris A., Sacco R. Ocular Fluid Dynamics. Anatomy, Physiology, Imaging Techniques, and Mathematical Modeling. New York: Springer-Birkhauser; 2019.

7. Ottesen JT, Danielsen M, editors. Mathematical modelling in medicine. Amsterdam: IOS Press; 2000. 
8. Layton AT, Edwards A. Mathematical Modeling in Renal Physiology. Springer; 2014.

9. Salerni F, Repetto R, Harris A, Pinsky P, Prud'homme C, Szopos M, Guidoboni G. Biofluid modeling of the coupled eye-brain system and insights into simulated microgravity conditions. PLoS One. 2019;14;14(8):e0216012.

10. Carichino L, Harris A, Guidoboni G, et al. A theoretical investigation of the increase in venous oxygen saturation levels in glaucoma patients. Journal for Modeling in Ophthalmology. 2016;1(1):64-87.

11. Carichino L, Guidoboni G, Verticchio Vercellin AC. Computer-aided identification of novel ophthalmic artery waveform parameters in healthy subjects and glaucoma patients. Journal for Modeling in Ophthalmology 2016;1(2):59-69.

12. Carichino L, Harris A, Lapin S. Waveform parameters of retrobulbar vessels in glaucoma patients with different demograpics and disease severity. Eur J Ophthalmol. 2019;7:1120672119848259.

13. Marquis AD, Arnold A, Dean-Bernhoft C, Carlson BE, Olufsen MS. Practical identifiability and uncertainty quantification of a pulsatile cardiovascular model. Math Biosci. 2018;304:9-24.

14. Bruttini C, Verticchio Vercellin AC, Montanaro B. The Mont Blanc Study - The effect of altitude on intraocular pressure and central corneal thickness in healthy volunteers. Invest Ophthalmol Vis Sci. 2018;59(9):1662. Abstract 1662. ARVO, April 29-May 3, 2018, Honolulu, HI.

15. Leete J, Layton AT. Sex-specific long-term blood pressure regulation: Modeling and analysis. Comput Biol Med. 2019;104:139-148.

16. Layton, AT. Optimizing SGLT inhibitor treatment for diabetes with chronic kidney diseases. Biol Cybern. 2019;113(1-2):139-148.

17. Ometto G, Assheton P, Calivá F, et al. Spatial distribution of early red lesions is a risk factor for development of vision-threatening diabetic retinopathy. Diabetologia. 2017;60(12):2361-2367.

18. Wang Y, Marron JS, Aydin LA, Bullitt E, Wang HA. Nonparametric regression model with tree- structured response. J Am Stat Assoc. 2012;107:1272-1285.

19. Arnold A, Calvetti D, Somersalo E. Parameter estimation for stiff deterministic dynamical systems via ensemble Kalman filter. Inverse Probl. 2014:30(10): 105008.

20. Shirinifard A, Glazier JA, Swat M,. Adhesion failures determine the pattern of choroidal neovascularization in the eye: a computer simulation study. PLoS Computat Biol. 2012;8(5):e1002440.

21. Zhu S, Shi F, Xiang D, Zhu W, Chen H, Chen X. Choroid neovascularization growth prediction with treatment based on reaction-diffusion model in 3-d oct images. IEEE J Biomed Health Inform. 2017;21(6):1667-1674.

22. Koeppen BM., Stanton BA. Berne and Levy Physiology. 7th edition. Elsevier; 2017.

23. Guidoboni G, Bonifazi, G, Sacco R, et al. Electrochemical characterization of ciliary epithelium physiology: a theoretical approach. Invest Ophthalmol Vis Sci. 2019; 60(9):3202. Abstract 3202. ARVO, April 28- May 2, 2019. Vancouver, Canada.

24. Stalmans I, Vandewalle E, Anderson DR, et al. Use of colour Doppler imaging in ocular blood flow research. Acta Ophthalmol. 2011;89(8):e609-30. doi:10.1111/j.1755-3768.2011.02178.x.

25. Hardarson SH, Basit S, Jonsdottir TE, et al. Oxygen saturation in human retinal vessels is higher in dark than in light. Invest Ophthalmol Vis Sci. 2009;50:2308-2311. doi:10.1167/iovs.08-2576.

26. Cassani S. Blood circulation and aqueous humor flow in the eye: multi-scale modeling and clinical applications. Ph.D. thesis, Purdue University 2016. 